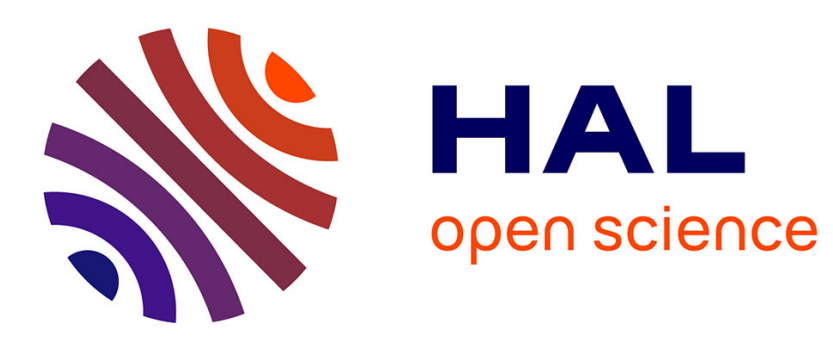

\title{
I-DIC-based identification strategy of failure criteria: application to titanium and nickel-based alloys
}

\author{
Dominik Lindner, Olivier Allix, François Hild, Xavier Pinelli, Olivier \\ Paulien-Camy
}

\section{- To cite this version:}

Dominik Lindner, Olivier Allix, François Hild, Xavier Pinelli, Olivier Paulien-Camy. I-DIC-based identification strategy of failure criteria: application to titanium and nickel-based alloys. Meccanica, 2016, 51, pp.3149-3165. 10.1007/s11012-016-0555-3 . hal-01480976

\section{HAL Id: hal-01480976 https://hal.science/hal-01480976}

Submitted on 2 Mar 2017

HAL is a multi-disciplinary open access archive for the deposit and dissemination of scientific research documents, whether they are published or not. The documents may come from teaching and research institutions in France or abroad, or from public or private research centers.
L'archive ouverte pluridisciplinaire HAL, est destinée au dépôt et à la diffusion de documents scientifiques de niveau recherche, publiés ou non, émanant des établissements d'enseignement et de recherche français ou étrangers, des laboratoires publics ou privés. 


\title{
I-DIC-based identification strategy of failure criteria:
}

\section{Application to Titanium and Nickel-based alloys}

\author{
Dominik LINDNER • Olivier ALLIX • \\ François HILD · Xavier PINELLI · Olivier \\ PAULIEN-CAMY
}

Received: date / Accepted: date

\begin{abstract}
Integrated Digital Image Correlation (I-DIC) is used to probe three different failure criteria on two high performance alloys. The approach consists of two steps. First, the mechanical state of each analyzed test is determined as best as possible by calibrating the parameters of a given elastoplastic law. Thin and thick samples are studied, which induce totally different states of equivalent plastic strain. Second, the state of stresses on the actual fractured surface by postprocessing the 3D finite strain simulations of the I-DIC framework. These data are
\end{abstract}

D. Lindner, O. Allix, F. Hild, X. Pinelli,

LMT, ENS Paris-Saclay/CNRS/Université Paris-Saclay

61 avenue du Président Wilson

F-94235 Cachan Cedex, France

E-mail: allix@lmt.ens-cachan.fr (corresponding author)

D. Lindner, O. Paulien-Camy

SAFRAN Helicopter Engines

avenue Joseph Szydlowski, F-64511 Bordes, France

E-mail: olivier.paulien@safrangroup.com 
used to analyze different local criteria. It is found that a maximum eigen stress criterion is well adapted to the studied materials.

Keywords Digital Image Correlation · Fractography · Finite Element simulations · Rankine criterion · Voce law

\section{Introduction}

The present study is motivated by the determination of the burst speed of turbomachine components made of Titanium and Nickel-based alloys. Such burst predictions may rely on global criteria such as the average hoop stress criterion proposed by Robinson [1]. In the case of complex geometries and refined material models, this criterion appears not to be accurate enough as experienced by SAFRAN Helicopter Engines. To overcome this limitation, today's trend is to rely instead on local burst predictions based on finite element simulations $[2,3]$. In such an approach two aspects are important, namely, the determination of trustworthy constitutive laws and the use of reliable damage or failure criteria. For both aspects, it is important to base the identification on tests whose states of plastic strain and stresses are representative of those occurring in service conditions.

To address these points, a preliminary study was conducted. It consisted of the determination of the level of stresses and plastic strains in actual disks by finite element simulations. Von-Mises elastoplastic model identified from previously performed uniaxial tests has been used [4]. Let us recall that prior to localization the stress state in these tests is characterized by a stress triaxiality of $\approx 0.33$. In the disk computations the stress triaxiality ranges from 0.4 to 0.8 [4]. Moreover large values of equivalent plastic strains are reached in the burst calculations. The val- 
ues lie in the post-peak regime of typical strain-stress curves in uniaxial tension.

In this regime, strain and stress heterogeneities occur in the tensile characteri-

zation test. This makes classical identification, which assumes test homogeneity, inappropriate.

This preliminary study led us to conduct experiments on thin and thick notched flat specimens, which under tensile loading, lead to stress and strain levels representative of those in actual disks $[5,4]$. The considered sample geometries induce non-uniform strain fields. In this case, the measurement of load-dependent displacement fields and their subsequent exploitation are necessary [6-8]. Different optical techniques can be used to measure displacement fields. Among them, Digital Image Correlation (DIC) is increasingly employed due to its versatility and applicability to "any" scale of observation [9].

Even though very powerful, DIC suffers from the 'resolution/spatial resolution' curse since the registration procedure is an inverse problem [10]. In particular, it does not allow elements of arbitrarily small sizes to be used. For some of the analyzed tests, this limitation occurred for a minimum element size of 60 pixels, due to the poor random pattern [5]. To correct for this shortcoming, it was decided to rely on Integrated DIC (I-DIC), which allows measurements and simulations to be linked in a seamless manner, and the element size to be tailored at will since the afore-mentioned limitation can be overcome. This technique was first applied with closed-form (i.e., elastic) solutions [11] and then generalized to numerically generated solutions [12-14]. In Ref. [5] this procedure was used on one thin sample made of Ti6Al4Va for which different elastoplastic postulates available in the commercial code Abaqus were compared. This study allowed an extended Voce law to be calibrated up to levels of equivalent plastic strain $\approx 40 \%$. These levels are 
far beyond the beginning of the post-peak regime in tensile tests on un-notched specimens, which is about $13 \%$ to $16 \%$ for the studied alloys [15]. Moreover, these levels of equivalent plastic strain were obtained for stress triaxialities ranging from 0.4 to 0.7 . Using the I-DIC procedure described in Ref. [5] these results are complemented by those obtained on one thick and three thin samples. The interest for the determination of failure criteria is that very different and much lower level of equivalent plastic strain are reached in thick samples. Another material will also be studied (i.e., nickel-based superalloy).

Early failure criteria of ductile materials mainly referred to yielding [16-19]. To experimentally probe such criteria, two different sample geometries have been considered. Bridgman used circular cylindrical bars and tubes to prove plastic incompressibility and that the yield stress was independent of the hydrostatic stress for various metals $[20,21]$. The initial yield locus and its subsequent changes were determined experimentally by Naghdi et al. [22] on thin tubes made of aluminum alloy subjected to combined tension and shear (i.e., torsion). To discriminate Tresca and von Mises criteria, Lode [23] applied combined tension and internal pressure on tubular samples. The author also discussed the role of the intermediate principal stress and introduced the now-called Lode parameter. Taylor and Quinney [24] carried on such studies on steel tubes by combining tension and torsion. Osgood et al. [25] added internal pressure to tension and torsion on tubular samples. More recently, Bocher and Delobelle [26] studied the behavior of 316L stainless steel under non-proportional loading conditions by combining tension, torsion internal and external pressure on tubular samples.

An alternative route to the previous studies is provided by biaxial experiments on sheets and plates (i.e., flat specimens [27]). This configuration is more chal- 
lenging since it requires more actuators (i.e., 4) than for tubular samples (i.e., typically 2). Another challenge is related to the evaluation of the stress field and its uniformity. Photoelasticity was used to check earlier designs [28]. Hayhurst [29] proposed a thinned geometry allowing creep rupture to occur in the central part of the sample. The designs were optimized over the years [30-34] to achieve the most uniform stress and strain states in the central part of such samples. Extensions to triaxial tests on volume elements have also been proposed [35-37]. However, their analysis is very delicate since the measurements cannot be performed very close to region of interest [38-41].

For flat samples, another route is to let heterogeneities develop and measure the corresponding kinematic fields. Metal forming has been an area in which punch tests have been considered $[42,43]$. One key aspect is the measurement of strains, which are visually determined by analyzing deformed grid or circle markings that are printed prior to the experiment [44-46]. Digital image correlation was introduced later on $[6,47]$ to analyze such tests. This last technique allowed the kinematic analysis to be fully automated. It will also be used herein when strongly coupled (i.e., integrated) with numerical simulations.

Using the identified extended Voce constitutive law it is possible to test different failure criteria with a large amount of data derived from I-DIC applied to both thin and thick samples. The principle is simple. The state of strain and stress is computed on the actual fracture surface of the specimen just prior to fracture. From these values different failure criteria are tested. The consistency of any proposed criterion can be checked by plotting the value at any point and time of the test to verify that the failure criterion was not reached earlier and/or at a different location for the conducted tests. If the principle seems simple its application is not 
straightforward. In fact the fracture of the specimen is sudden. This means that from one recorded image to the next the specimen has broken. Therefore an issue is how to recover from two broken parts the actual positions of the crack in the unbroken specimen. It is then needed to locate this surface within the finite element mesh to recover the state of strains and stresses by a post-processing technique.

The outline of the paper is as follows. The two studied materials and the two sample geometries are introduced in Section 2. Section 3 deals with the procedure of determining the mechanical state of the tests at the onset of fracture. Fractographic analyses and the fracture loci are then discussed for a titanium alloy in Section 4 and a nickel-based superalloy in Section 5 .

\section{Studied Materials and Experiments}

\subsection{Studied Materials}

Because of their use in helicopter engines, this study examines the titanium alloy Ti6Al4V or TA6V and the nickel-based super alloy NiCr19Co18Mo4Ti3Al3 or Udimet 500 (U500). Their actual microstructure strongly depends on the heattreatment. Examples of the microstructures of the two alloys are displayed in Figure 1. The alloy composition and the final morphology of the grains determine the mechanical properties $[48,49]$.

2.1.1 Titanium-based TA6V alloy

The titanium alloy Ti6Al4V (hereafter designated as TA6V) is a standard aeronautical material with widespread use in structural parts of planes and engines. TA6V combines several titanium phases. The two main phases are a hexagonal 


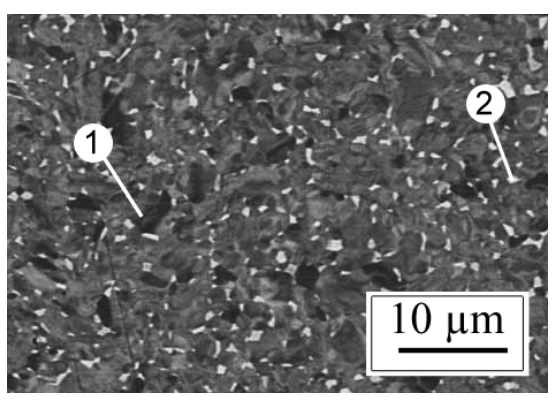

(a) TA6V

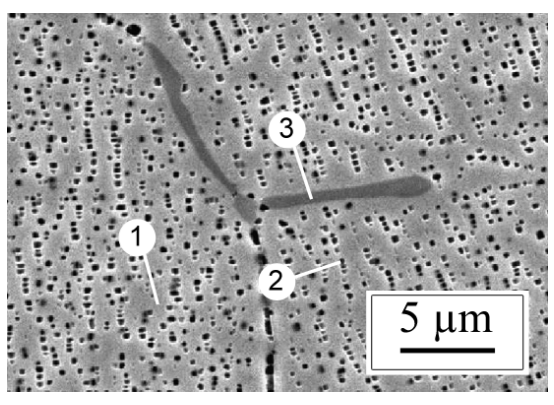

(b) U500

Fig. 1 Microstructure of the studied alloys. (a) TA6V: (1) $\alpha$-phase (dark structures) is filled with (2) $\beta$-phase (white patches), after Ref. [48]. (b) U500: (1) $\gamma$-phase (light gray) is filled with (2) $\gamma^{\prime}$-phase (black), (3) carbides exist (dark gray), after Ref. [49]

$\alpha$-phase and a body centered cubic $\beta$-phase (Figure 1(a)). Additional elements provide stability and corrosion resistance to these phases. Table 1 presents an overview of the alloy elemental composition. Depending on the heat-treatment, the typical mechanical properties are characterized by a Young's modulus ranging from $E=110$ to $140 \mathrm{GPa}$, a Poisson's ratio $\nu=0.32$, an ultimate stress $\sigma_{\max }$ from 900 to $1200 \mathrm{MPa}$ and a "macroscopic" elongation to failure $\epsilon_{\max }$ measured in tensile tests from 13 to $16 \%$ [15].

\subsubsection{Nickel-based Udimet 500 superalloy}

The increased temperature and corrosion resistance of the nickel-based Udimet 500 alloy is associated with the higher amount of chromium and cobalt. In a typical composition, these add up to $35 \%$ of the total weight (Table 1 ). The material consists of a primary body centered cubic $\gamma$-phase, and a strengthening face centered cubic $\gamma^{\prime}$-phase. The large quantity of additional alloying elements and higher amounts of carbon lead to a significant formation of carbides [50], see Figure 1(b). Similar to TA6V the large variety of heat-treatments leads to a 
large range of mechanical properties, namely, an elastic behavior with a Young's modulus $E \approx 200 \mathrm{GPa}$ and a Poisson's ratio $\nu=0.3$. The ultimate tensile strength $\sigma_{\max }$ ranges from 690 to $1300 \mathrm{MPa}$ and a "macroscopic" elongation to failure $\epsilon_{\max }$ from 4 to $18 \%[51]$.

Table 1 Alloy composition of most important elements in weight percent (\%), after Ref. [15] for TA6V, and Ref. [49] for U500

\begin{tabular}{|c||c|c|c|c|c|}
\hline Alloy & Al & $\mathbf{C}$ & $\mathbf{C o}$ & $\mathbf{C r}$ & $\mathbf{F e}$ \\
\hline TA6V & 5.91 & 0.02 & - & - & 0.11 \\
\hline U500 & 3.0 & 0.11 & 17.2 & 17.9 & 2.1 \\
\hline Alloy & Mo & $\mathbf{~ N i}$ & $\mathbf{O}$ & $\mathbf{T i}$ & $\mathbf{V}$ \\
\hline TA6V & - & - & 0.12 & Base & 3.85 \\
\hline U500 & 4.0 & Base & - & 3.0 & - \\
\hline
\end{tabular}

2.2 Sample Geometries and Test

Stress triaxialities representative of turbo-engine applications vary within the range $0.4 \leq \eta \leq 0.75$ [4], where $\eta=\sigma_{h} / \sigma_{e q}$ with $\sigma_{h}$ the hydrostatic stress and $\sigma_{e q}$ von Mises' equivalent stress. Several geometries allow for testing such ranges, namely, round notched samples, flat samples with notch or hole and biaxial samples. In order to master as well as possible the loading conditions, only uniaxial tests are considered. The use of 2D-DIC requires flat surfaces or a very small curvature. To minimize (and possibly eliminate) the spurious effects associated with out-of-plane motions telecentric lenses have systematically been used in the experiments reported hereafter. Only flat geometries were considered. Within these 
geometries, notches allow for higher stress triaxialities than holes. With the employed equipment, the sample maximum cross-section was set to $8 \times 7 \mathrm{~mm}$.

Taking these considerations into account led to the design of a 7-mm thick sample. In order to test the procedure on a sample with lower three-dimensional effects and possibly higher strains it was also decided to use thin (i.e., 0.7-mm thick) samples. Figure 2 depicts the final designs.

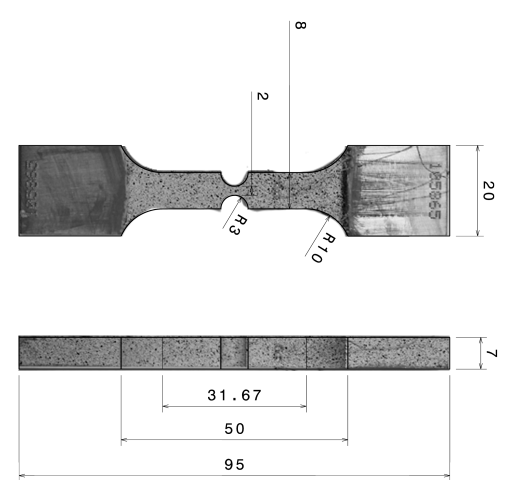

(a) 7-mm thick sample

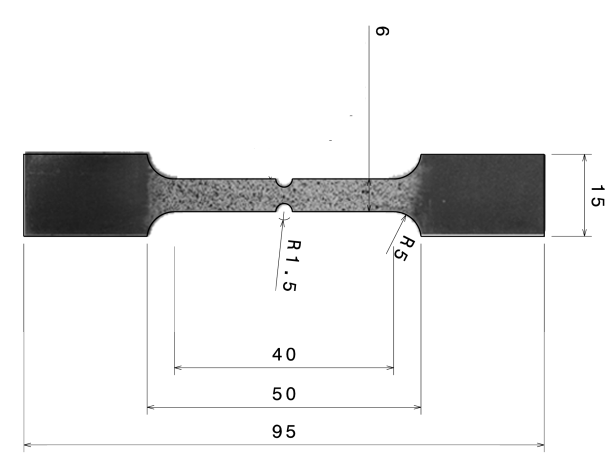

(b) $0.7-\mathrm{mm}$ thick sample

Fig. 2 Sample geometries (dimensions in $\mathrm{mm}$ ). Both samples are displayed with examples of the speckle pattern; the gripped area is kept paint-free to avoid slip

The tests were conducted in uniaxial tension with a $100-\mathrm{KN}$ servohydraulic machine. To perform these tests as far as possible (i.e., under stable condition) they were displacement-controlled. The inter-grip strain rate was set to $\dot{\epsilon}=0.001 \mathrm{~s}^{-1}$. In addition, some samples were subjected to unloading-reloading cycles to study potential evidence of damage.

The samples were fabricated from extruded bars via electric discharge machining. In a last manufacturing step, the notch section of the samples was polished to avoid fracture due to surface defects. The two principal directions (i.e., longitudinal (L) and transverse (T)) of the bar were tested to account for possible anisotropy. 
Samples of every possible combination of material (TA6V, U500), thickness (i.e., 0.7 and $7 \mathrm{~mm}$ ) and direction (L, T) were tested one or three times.

The polishing of the surfaces induced a non-optimal adhesion of the speckle pattern paint [5]. The modification of the standard preparation method allowed the problem to be circumvented. The iterative application of black and white droplets with intermediate pauses promoted a better adhesion of the paint. A resulting disadvantage was a heterogeneous pattern quality.

\section{Evaluating Stress States at Failure Onset}

\subsection{Implemented Procedure}

In the sequel, Integrated DIC will be used to directly evaluate mechanical fields to extract any quantity of interest. To have access to such fields, the parameters of the chosen constitutive law have to be known. They are part of the proposed procedure, which calibrates them to get the most reliable estimates for the given constitutive model. Since various tests and geometries will be considered, the calibration is performed independently for each single experiment.

To determine the location of the fracture surface, an imaged-based analysis is performed. It consists in registering the first image of the broken sample with respect to the last one prior to fracture. The elements that are cut by the crack are then extracted from the initial mesh. Fracture loci can then be determined by processing the finite element data. In the sequel, the mechanical state is evaluated for the instant just before fracture. 
3.2 Mechanical State of Tested Samples

To evaluate the mechanical state of tested samples, the constitutive law should be known. Once a constitutive model is chosen, the corresponding material parameters need to be calibrated. The identification of material parameters will be based upon I-DIC.

First, global DIC consists of measuring displacement fields between a gray level picture $f$ in the reference configuration and a series of pictures $g$ in the deformed configuration [52]. The sum of squared gray level differences is minimized for each time $t$ over the whole region of interest (ROI) $\Omega$

$$
\chi_{c}^{2}(t)=\frac{1}{2 \gamma_{f}^{2}|\Omega|} \sum_{\Omega}(g(\mathbf{x}+\mathbf{u}(\mathbf{x}, t), t)-f(\mathbf{x}))^{2}
$$

with respect to the parameterization of the measured displacement field $\mathbf{u}(\mathbf{x}, t)$, where $\mathbf{x}$ is any pixel of the ROI $\Omega, t$ the considered time, and $\gamma_{f}^{2}$ the variance of image acquisition noise that is assumed to be white and Gaussian. In the following, the parameterization of the displacement field is based upon 4-noded quadrilaterals of an unstructured mesh that describe the external surfaces of 3D finite element models, which are made consistent with the experimental configuration. This first measurement technique was used to assess the model quality [5] and also provided the Dirichlet boundary conditions that will be used to drive finite element simulations.

Let $\{\mathbf{p}\}$ denote the column vector gathering all unknown material parameters. I-DIC consists of globally minimizing over space and time the total identification residual with respect to the sought material parameters

$$
\left\{\mathbf{p}^{\mathrm{opt}}\right\}=\underset{\{\mathbf{p}\}}{\operatorname{argmin}} \chi_{\text {tot }}^{2}(\{\mathbf{p}\})
$$


with

$$
\begin{aligned}
\chi_{\mathrm{tot}}^{2} & =\frac{1}{2} \chi_{c}^{2}+\frac{1}{2} \chi_{F}^{2} \\
\chi_{c}^{2} & =\frac{1}{n} \sum_{n} \chi_{c}^{2}(t) \\
\chi_{F}^{2}(\{\mathbf{p}\}) & =\frac{1}{n \gamma_{F}^{2}}\left(\left\{\mathbf{F}_{\mathbf{m}}\right\}-\left\{\mathbf{F}_{\mathbf{c}}(\{\mathbf{p}\})\right\}\right)^{t}\left(\left\{\mathbf{F}_{\mathbf{m}}\right\}-\left\{\mathbf{F}_{\mathbf{c}}(\{\mathbf{p}\})\right\}\right)
\end{aligned}
$$

where $\chi_{F}^{2}$ denotes the global equilibrium gap, which is to be minimized in conjunction with the global correlation residuals $\chi_{c}^{2}$. Vector $\left\{\mathbf{F}_{\mathbf{m}}\right\}$ gathers all measured load levels for all considered time steps $n$, and $\left\{\mathbf{F}_{\mathbf{c}}\right\}$ all computed reaction forces. $\gamma_{F}$ denotes the standard resolution of the load measurement. A Gauss-Newton algorithm is implemented to minimize $\chi_{\text {tot }}^{2}$, which is nonlinear with respect to $\{\mathbf{p}\}$. Forward finite differences are considered to compute the sensitivity fields for the minimization procedure with the commercial FE code Abaqus implicit. This type of procedure utilizes any FE code, be it commercial, in a non intrusive way [14]. 3D FE simulations are run with reduced integration elements with hourglass stabilization to prevent from volumetric locking. It is worth noting that with the present technique, the mesh can be made as fine as needed since the number of unknowns has been significantly reduced to the number of sought material parameters instead of the nodal degrees of freedom in Q4-DIC. This in turn provides a strong robustness of the whole procedure and allows imperfections such as cracking of the paint and poor quality of the speckle pattern to be dealt with effectively [5].

In I-DIC approaches, one important feature is the choice of the constitutive law. In the present case, elastoplasticity is modeled with J2-flow rule and isotropic hardening described by extended Voce law (i.e., exponential + linear hardening) for which the yield criterion reads

$$
\sigma_{e q}=\sigma_{y}+C p+\sigma_{0}\left(1-\exp \left(p / p_{0}\right)\right)
$$


where $p$ denotes the cumulated plastic strain, $\sigma_{y}$ the yield stress, $\sigma_{0}$ the hardening stress, $p_{0}$ the hardening strain, and $C$ the linear hardening modulus. It was shown to be the closest to an earlier experiment on one of the studied materials [5]. The calibration of Poisson' ratio $\nu$ turned out to be delicate. The reference value for each material was set. Consequently, for extended Voce law, five parameters are tuned (i.e., Young's modulus $E$, yield stress $\sigma_{y}$, hardening stress $\sigma_{0}$, hardening strain $p_{0}$, and linear hardening modulus $C$ ).

\subsection{Image-Based Analysis of Cracked Surface}

The image after fracture cannot be analyzed by the current I-DIC procedure, as the sample is broken. However, it is possible to correlate one part of the broken sample with the preceding image, that is, the last image of the I-DIC analysis. The final displacement field is mostly limited to elastic unloading and a large rigid body motion. The rigid body motion results from the feedback response of the testing machine as the force quickly drops to zero. This post-fracture displacement field could be identified by the restriction of a DIC analysis to one half of the sample.

To demonstrate the principle of the selection process, this is not necessary and such a DIC procedure was discarded. The elastic unloading was neglected and only the rigid body motion was identified. The last image was shifted, until the upper part of the broken sample corresponded globally to the location of the unbroken sample in the previous image (Figure 3(a)). The data of the last I-DIC step were superimposed onto this shifted image of fracture (Figure 3(b)). As the upper part was used for manual correlation, the mesh and the recorded sample limits in the lower part do not correspond to each other. 


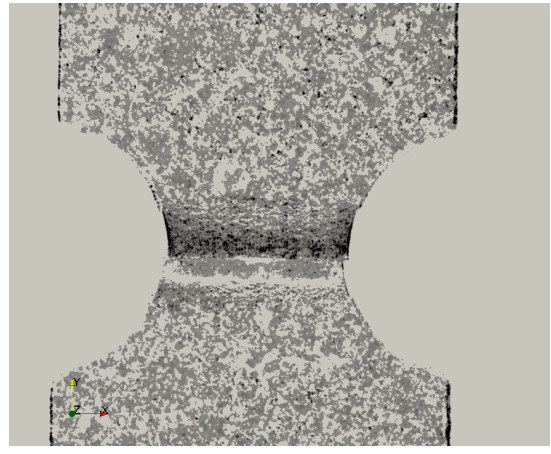

(a)

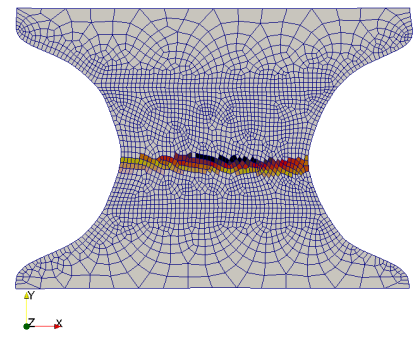

(c)

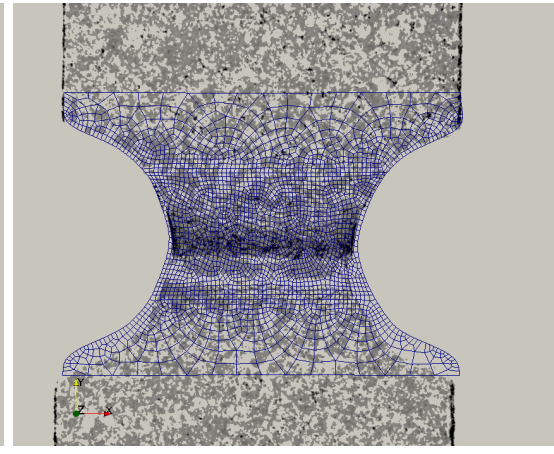

(b)

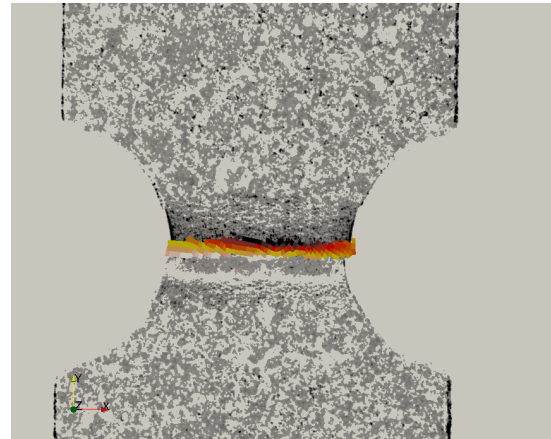

(d)

Fig. 3 Selection procedure for the fracture locus for a thin sample made of TA6V. (a) Broken sample. (b) Superposition of broken sample and I-DIC result before fracture. (c) Selection of elements in the critical zone. (d) Superposition of the equivalent plastic strain in the selected zone with the fracture image

In the upper part, the superposition allows for the selection of the elements that are closest to the fracture surface. The selected elements in this critical zone are shown in Figure 3(c), and they were extracted from the mesh (Figure 3(d)). The color map indicates the equivalent plastic strain within the elements; a darker color symbolizes a higher value. 
For the thick sample the same procedure was performed, see Figure 4. The selected zone depends on the actual test conditions. The fracture surface can vary in the out-of-plane direction. However, for 3D simulations the boundary conditions were a simple extrusion of the face boundary conditions. Therefore, the same procedure was applied for the 3D fracture surface. All elements that are an extrusion of the surface selection were extracted from the 3D model.

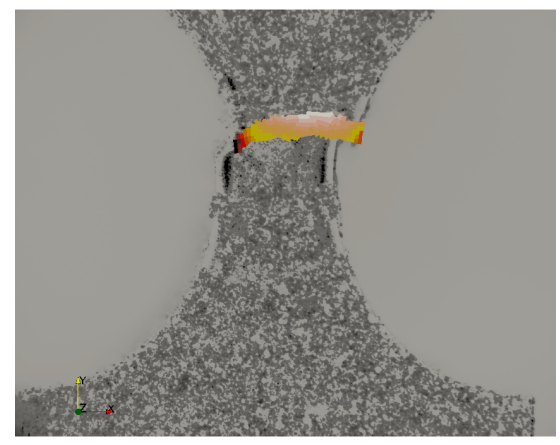

Fig. 4 Superposition of shifted fracture image and critical elements for the thick sample made of TA6V

\subsection{Construction of Fracture Loci}

The quantities that are used for the prediction of fracture vary for existing criteria. They are either stress, strain [53] or energy-based [54]. Among the stress-based quantities are the different equivalent stresses, as for example von Mises $[55,17$, 56], Tresca [16] or Coulomb [57] stresses. The maximum principal stress (or Rankine [58] criterion) is frequently used to describe brittle fracture. Finally the stress invariants are used in more complex fracture criteria. The strain-based quantities are associated with these stresses. The most commonly employed quantity is 
the equivalent plastic strain, which is used for ductile materials [59-61]. Other quantities are the principal strains [53].

A particular case is the use of damage criteria. Damage can be driven by the state of stress and strain. For ductile damage, it is possible that the stress triaxiality increases the amount of damage that exists for a certain equivalent plastic strain. For the Lemaitre damage law [62], the influence is described by the stress triaxiality function $R_{\nu}$. Due to the uncertainty on the role of damage a simplified damage indicator $D_{i}$ is proposed, which weights the equivalent plastic strain with the stress triaxiality function,

$$
D_{i}=p R_{\nu}
$$

Different propositions were tested [4] and only one will be reported herein, namely,

$$
R_{\nu}=\left(1+\beta_{D}\left[\eta^{2}-(1 / 3)^{2}\right]\right)
$$

with $\beta_{D}=6$.

\section{Results for TA6V Alloy}

This section reports the results obtained for six thin notched samples and one thick notched specimen made of TA6V alloy.

\subsection{Hardening Response}

The extended Voce law was identified on all thin samples. As the identified hardening curves are not identical, an average curve was calculated from the test data. This result is illustrated for the longitudinal direction in Figure 5(a). For each 
sample, both planar faces were analyzed independently with the pictures acquired by cameras $\mathrm{C} 1$ and C4. Due to the non-linearity of the laws, the mean fit curve is calculated from the six curves and not from the coefficients of the curves. The maximum equivalent plastic strain is set to the average of all six analyses. The mean global residual level is of the order of $\bar{\chi}_{t o t}=7$, which is considered very low given the complex geometry of the sample and the test conditions [5].

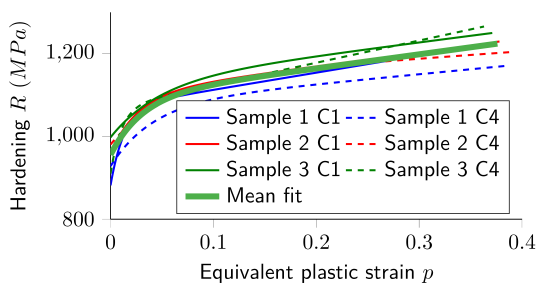

(a) L-direction

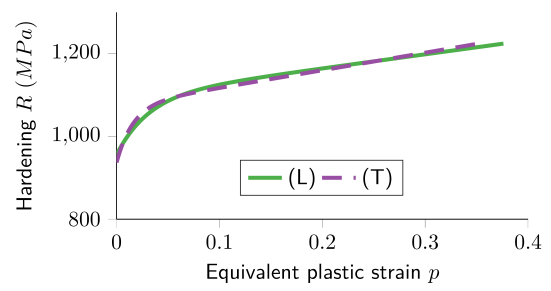

(b) Both directions

Fig. 5 Hardening curves from I-DIC analyses for thin samples made of TA6V. (a) Hardening curves for all analyzed cases in the longitudinal direction. (b) Average hardening curves for both directions

The average material behavior is identical for both tested directions, see Figure 5 (b). When $p=0.1$, the predicted hardening stresses are $\bar{R}(p=0.1)_{L}=$ $1110 \mathrm{MPa}, \bar{R}(p=0.1)_{T}=1130 \mathrm{MPa}$. The relative differences between the directions were of the same order as the variation for samples of the same direction. Similar observations were made for the maximum equivalent plastic strain, $\bar{p}_{\max _{L}}=0.39, \bar{p}_{\max _{T}}=0.38$.

The analysis of the thick sample was also performed. The most important point, which will be discussed later on, is the difference in failure strains. The mean value was $\bar{p}_{\max _{L}}=0.17$. This type of effect is expected since thick samples 
lead to higher stress triaxialities, and thus the failure strain is expected to be lower $[62,63]$.

The identification residuals of the thick samples were on average higher. For the example presented hereafter, the value was $\chi_{\text {tot }}=25$ (in comparison to $\bar{\chi}_{\text {tot }}=7$ for thin samples). This means that the results as such are less trustworthy than for the thin samples. However, the calculated forces from the identification procedure are still close to the experimental forces (Figure 6). The abscissa shows the loading parameter, $\epsilon_{\text {macro }}$, which corresponds to the mean longitudinal strain of the ROI. To assess the difference between thin and thick samples, the average hardening law of thin samples was also considered to analyze the data of a thick sample. This step will be referred to as verification. For the chosen example the verification residual rose to $\chi_{\text {tot }}=160$. There is a clear degradation of the overall quality. In addition, the forces are significantly lower.

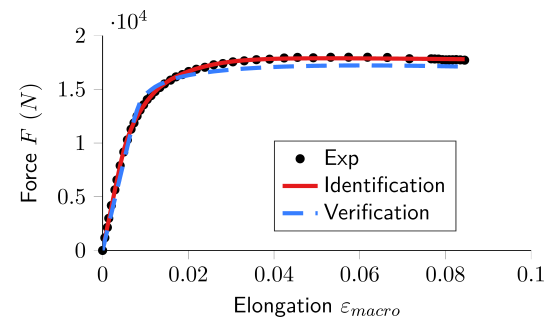

Fig. 6 Force-elongation curves for a thick sample for its identification and verification 
4.2 Fracture of TA6V

\subsubsection{Microscopic analysis}

An important step in the analysis of the materials is the characterization of the fracture surface. The examination of the broken sample surface was performed with scanning electron microscopy. Figure 7 shows an overview of the fracture surface and a zoom. The presented examples are representative of thin and thick samples. The fracture surface is not flat and resembles the cup cone structure,

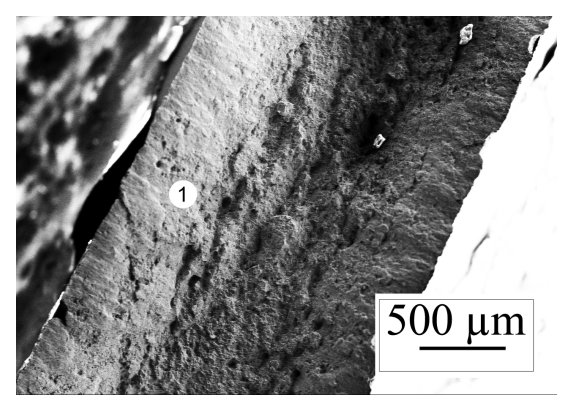

(a)

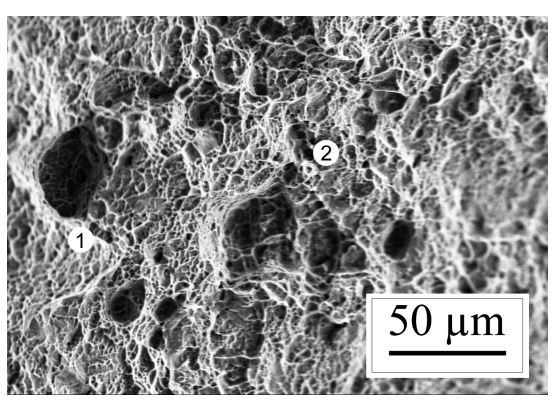

(b)

Fig. 7 Fractographies of notched samples made of TA6V. (a) Entire fractured surface; (b) a zoom reveals larger voids (1) and dimples (2)

which occurs in ductile materials. On a closer inspection of the surface, two distinguishable features are revealed. Considerably larger voids exist on the surface, i.e., at point (1). The size of the pores reaches almost $25 \mu \mathrm{m}$. A possible explanation is the debonding of grains or inclusion particles. In addition, repetitive structures cover the entire surface at point (2). These features are dimples typically observed in ductile rupture. Therefore, the fracture may be described as predominantly transgranular. These observations indicate that the final fracture mechanism of TA6V corresponds to the definition of ductile rupture. 


\subsubsection{Mesoscopic analysis}

Ductile rupture is the result of void nucleation and growth. If substantial and diffusive amount of damage occurs in the tested sample, the global elastic stiffness allows the damage changes to be identified. If such a method is commonly used for composite materials [63], such a global decrease in the apparent modulus of elasticity is less documented in the literature for metallic materials [62]. However, the DIC and especially I-DIC procedures have proven to be sensitive to small changes unseen with other techniques by using correlation residuals [5].

To study possible occurrence of damage with the used testing equipment, several unloading and reloading cycles were included for a thin sample. In a first step, the identification of the optimal elastoplastic parameters was performed for an image selection of continuously growing elongation, excluding the cycles. In a second step, the model parameters were used on the entire test data, including the cycles. The entire force-elongation curve is well described including the macroscopic softening in addition to the cycles, see Figure 8(a).

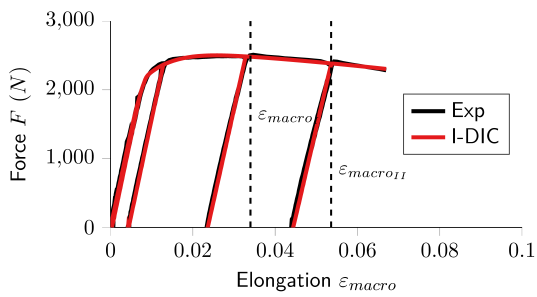

(a)

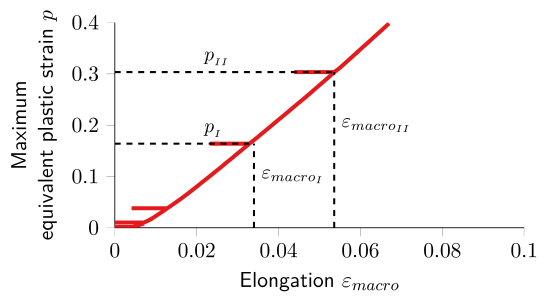

(b)

Fig. 8 Results from loading-unloading test. (a) Force-elongation curve and (b) I-DIC maximum equivalent plastic strain 
Figure $8(\mathrm{~b})$ shows the maximum equivalent plastic strain that was reached at every macroscopic elongation in I-DIC. The instants of the last two unloadings are marked in both diagrams with $\epsilon_{\text {macro }}=0.032$ and $\epsilon_{\text {macro }}=0.053$. At these instants, the equivalent plastic strain is $p_{I}=0.163$ and $p_{I I}=0.303$. The absence of significant deviation of I-DIC from the experiment means that the global effect of damage is not detected at $p_{I I}$. Due to the strong heterogeneity of the test, this does not imply that no local damage exists in the specimen for these values of equivalent plastic strain. Additional cycles after $\epsilon_{\text {macro }}$ II could deliver more precise information. However, this would require a higher cycle frequency. In the performed test campaign, this was avoided due to the increased likelihood of paint cracking [5]. Due to the lower level of plastic strain in the thick sample compared to the thin ones, no visible global effect of possible damage was expected prior to failure. Therefore, no cyclic tests were performed on thick samples.

Instead of analyzing the possible damage behavior, the present study focuses on fracture initiation. It seems that the damage nucleation and growth phases, which could precede TA6V fracture, can be neglected in a first instance, as it occurs very late and for a very short duration. This type of observation was also reported for aluminum alloys (see, e.g., Ref. [64]). The fact that thin and thick samples fail for very different values of the equivalent plastic strain led to the investigation of various criteria, notably those involving the effect of stress triaxiality.

\subsubsection{Fracture loci}

Once the state of strains and stresses "on" the fracture surface is known at an instant as close as possible to the initiation time, it is possible to create any possible combination for a fracture locus. The most common representation describes the 
maximum equivalent plastic strain $p$ over a range of stress triaxialities $\eta$. The values of the selected elements can be plotted in a fracture locus (Figure 9(a)). The graph contains the data points of thin and thick samples. Despite the tremendous reduction of data, the states of stress and strain are still heterogeneous.

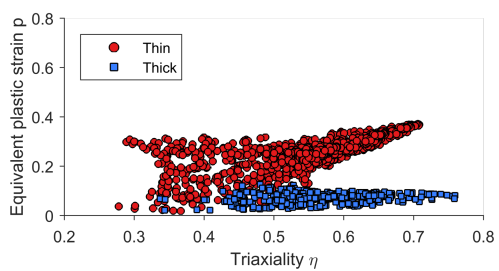

(a)

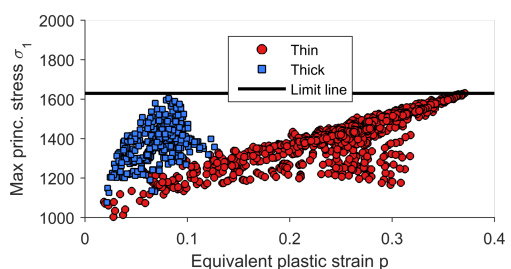

(b)

Fig. 9 Fracture loci $p(\eta)$ (a) and $\sigma_{1}(p)$ (b) for the elements in the critical section of TA6V samples

For the thin sample, the location of maximum equivalent plastic strain $p$ and maximum stress triaxiality $\eta$ coincide and it seems very likely that this combination leads to fracture initiation. For the thick sample the plastic strain is almost uniform in the ROI over the entire triaxiality range. The "maximum" equivalent plastic strain is reached when $\eta=0.48$. However, this state cannot induce fracture, as it also exists in thin samples. It seems therefore more likely that failure is associated with the strains at the highest stress triaxiality.

The definition of a criterion is difficult in this case. If the two points were to be connected by a line this would indicate that the material fails almost at a critical stress triaxiality. This contradicts the findings in the literature, namely, ductile materials usually show a very distinctive dependence of the maximum equivalent plastic strain $p$ on triaxiality $[62,63]$. This fracture locus does not seem to be appropriate for the reported tests on TA6V samples. 
Another proposition for a fracture locus is the use of the maximum principal stress $\sigma_{1}$ and equivalent plastic strain $p$ (Figure $9(\mathrm{~b})$ ). In contrast to literature results, no influence of the level of the equivalent plastic strain is found. A limit line can be drawn for $\sigma_{1}=1630 \mathrm{MPa}$. The fracture of TA6V could only be sensitive to the maximum principal stress, given that the computation should consider plasticity and large strains.

For ductile damage, it is possible that the stress triaxiality increases the amount of damage that exists for a certain equivalent plastic strain. Compared to the equivalent plastic strain $p-$ stress triaxiality $\eta$ locus, the function $R_{\nu}$ increases the importance at high strains. For both samples the maximum damage level correlates with the maximum stress triaxiality. However, there is no consistency between thin and thick samples (Figure 10). Consequently, the only compatible criterion corresponds to a maximum principal stress independent of the equivalent plastic strain level for TA6V.

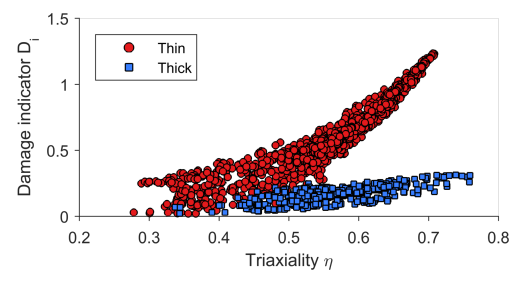

Fig. 10 Fracture locus for the damage indicator $D_{i}$ vs. stress triaxiality $\eta$ for elements in critical section for TA6V

\subsubsection{Validation of fracture locus}

The final step in the proposed procedure is the evaluation of the fracture locus for the entire data set. Figure 11 shows this result for the last step of the analysis. It 
was checked that the final step leads to higher values than the preceding ones [4]. For the maximum principal stress-equivalent plastic strain locus, all points are below the limit line. This means the selected elements contained the fracture surface. The same conclusion was drawn for the other investigated loci.

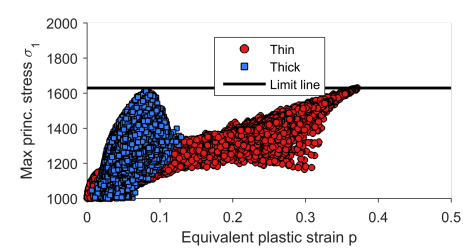

Fig. 11 Verification of the fracture locus in the $\sigma_{1}-p$ domain for all elements in the last step of the analysis for TA6V samples

\section{Characterization of U500 Alloy}

In the following, the results obtained for six thin notched samples and one thick notched specimen made of U500 are discussed.

\subsection{Hardening Response}

The mean identification residual for thin samples was of the order of $\bar{\chi}_{t o t}=9.2$ for the extended Voce law. This level is close to that observed for TA6V samples (i.e., $\bar{\chi}_{t o t}=7$ ). Consequently, the identification quality is similar for thin samples irrespective of the material differences. Figure 12(a) shows two examples of the force-elongation curves. The thin sample in the transverse direction broke at a lower strain. In fact, this result was confirmed for all samples in that direction. The average hardening curves are presented in Figure 12(b). 


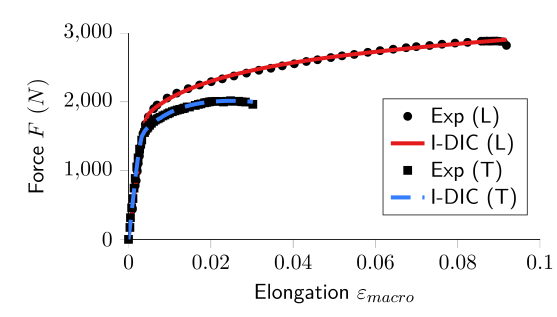

(a)

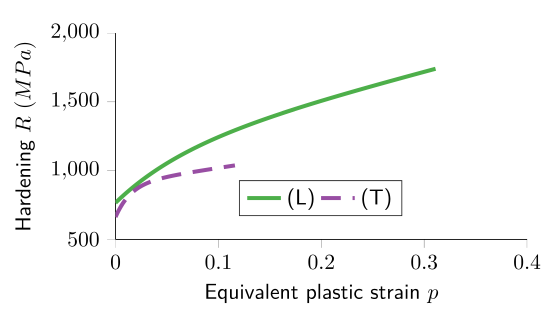

(b)

Fig. 12 I-DIC results for thin samples made of U500. (a) Force-elongation curves for two thin samples in both directions. (b) Average hardening curves for both directions

For the longitudinal direction, the average maximum equivalent plastic strain is $\bar{p}_{\max _{L}}=0.31$, whereas for the transverse direction only an average of $\bar{p}_{\max _{T}}=0.12$ was obtained. This is about $39 \%$ of the longitudinal value. In addition, the levels of stress in the transverse direction are significantly lower, $\bar{R}(p=0.1)_{L}=1240 \mathrm{MPa}$, $\bar{R}(p=0.1)_{T}=1020 \mathrm{MPa}$. This indicates that the transverse direction could be as much as $18 \%$ weaker. In the present case, there is a more pronounced anisotropy of the behavior when the longitudinal and transverse responses are compared.

The same procedure as for TA6V was applied to identify the difference between thin and thick samples. As for TA6V, the increase in thickness led to a lower maximum equivalent plastic strain, $\bar{p}_{\max _{L}}=0.18$. This level is lower than that observed in the thin samples. This effect can be explained by the stress triaxiality level that is different in thin and thick samples.

For the thick sample used in the following, the total identification residual was $\chi_{\text {tot }}=27$. The verification residual rose to $\chi_{\text {tot }}=450$, a value significantly higher. There is a strong effect between the two sets of material parameters on the identification quality, which is also visible on the force-elongation curve (Figure 13). The difference between identification and verification forces is higher for U500 


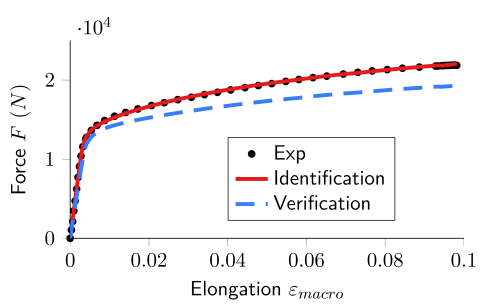

Fig. 13 Force-elongation curve for a thick sample for its identification and the verification with the average model of the thin samples made of U500

than TA6V. To examine the possible origin of such a difference, the hypothesis of the extraction of the boundary conditions was verified on an FE model of the entire sample geometry. The sample was modeled once completely and once only as a section of $4 \mathrm{~mm}$ in length, similar to the extent of the used ROI in I-DIC (Figure 14(a)). The nominal geometry and the identified mean constitutive model parameters of the thin samples were used. Mesh constraints were applied to ensure the mesh of the ROI corresponds to that in the same region of the complete model. In the case of the complete model, the sample was loaded uniformly in tension at its upper end, marked with (1), and fixed at the lower end, point (2). This loading leads to similar displacements on the ROI boundary as in the experiment and is therefore denominated as "true" boundary condition (BC). After the calculation of the model the longitudinal displacement at the limits of the ROI were extracted on the surface, point (3), and a 4th order polynomial was fitted to these data. The obtained field was then applied as loading condition to the boundary surface nodes of the ROI model and extruded for all nodes in the thickness direction; a procedure that is assumed to be almost equivalent to the processing of the experimental data in I-DIC. 


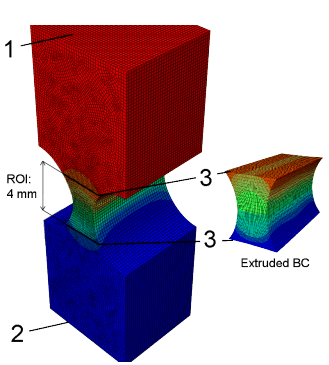

(a)

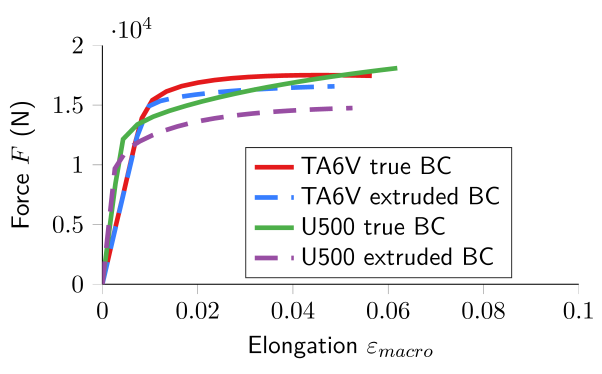

(b)

Fig. 14 Verification of boundary condition extrusion hypothesis. (a) The sample is modeled completely and as a model of the ROI. (b) Force-elongation curves from the two models

The mean displacement of the ROI boundary and the associated macroscopic elongation were calculated for both models (true and extruded BCs). Figure 14(b) shows the force-elongation curves. For the ROI model with the extruded boundary conditions the forces are lower. As in the verification step, the differences between true and extruded boundary conditions are larger for U500 compared to TA6V alloy. This leads to the following explanation. In the I-DIC identification, the lower forces were compensated by the stress level of the material model, which was higher for the thick samples compared to the thin samples.

The difference in the resulting errors between TA6V and U500 can be explained as follows. U500 has a lower yield stress and a stronger hardening modulus. This results in a greater extent of the plasticity field. In addition, the plastic strain field is not uniform in the thickness. For a similar size of the ROI, the experimental displacement boundary surface is then more deformed in the thickness compared to TA6V and the assumption of the extrusion is less correct.

To improve the accuracy, more elaborate procedures are necessary to deal with these kinds of boundary conditions. One solution is the enlargement of the ROI. However, this was difficult as the strong notch curvature and the resulting 
element shape made DIC impossible for refined meshes [5]. An improvement could be achieved through the interpolation from separate coarse DIC solutions to fine boundary distributions for I-DIC. Another solution would be the introduction of the boundary conditions to the set of unknowns $[65,66]$ in I-DIC approaches. In that case DIC analyses would not be required.

\subsection{Microscopic and Mesoscopic Analyses}

The previous methodology was applied to the U500 test results. The examination of the fracture surface clearly reveals the structure of the grains (Figure 15). In addition, the macroscopic force-elongation curve for any sample showed no significant softening. Both results are as expected for brittle fracture. Therefore, no cyclic tests were performed. Yet, the fracture surface is not straight. Larger pores mark the surface, i.e., close to the notch at point (1). The occurrence of bands with distorted microstructure is the microscopic cause of the mentioned macroscopic anisotropy (2). On closer inspection of the grains, a coarse texture, which covers the entire grain surface, is found (3). The most likely explanation is the existence of microvoids on the grain boundaries.

\subsection{Construction of the fracture loci}

The selection of the elements in the vicinity of the fracture surface was performed analogously to that for TA6V samples (Figure 16).

The same fracture loci as for TA6V were evaluated. Even though the material fails in a brittle manner, the fracture locus usually employed for ductile rupture, namely, equivalent plastic strain $p$ - stress triaxiality $\eta$, is remarkable. For thin 


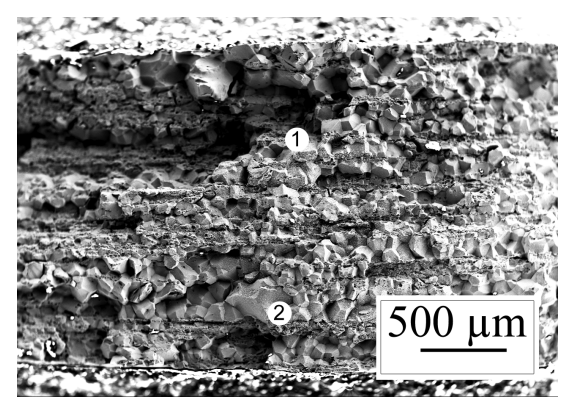

(a)

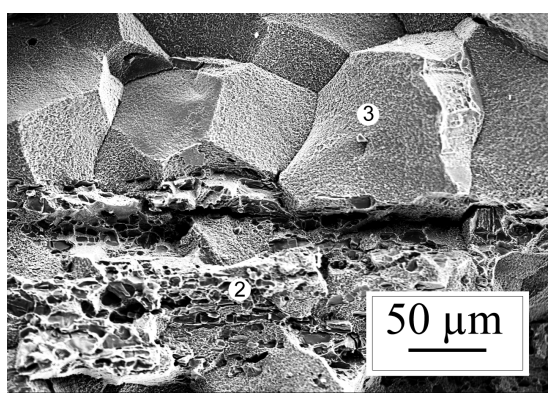

(b)

Fig. 15 Fractographies of notched samples made of U500. (a) Entire intergranular fracture with complex surface structure (1). (b) Zoom with distortion bands (2) and microvoids on the grain boundary (3)

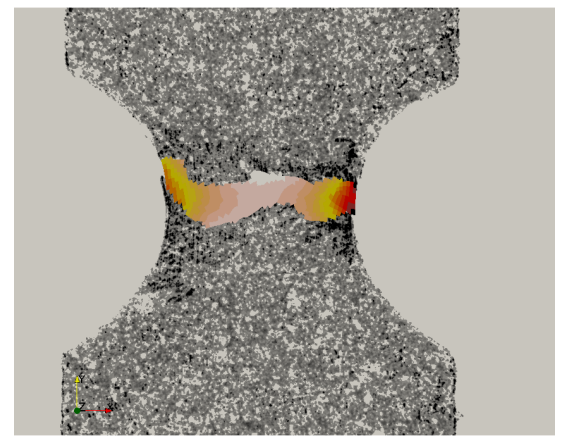

(a) Thin sample

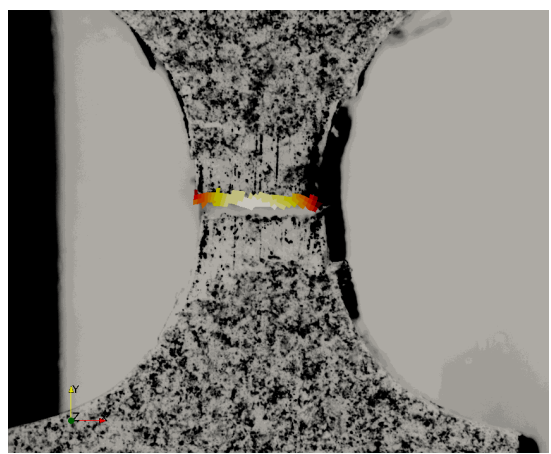

(b) Thick sample

Fig. 16 Selections of the critical elements for two samples made of U500

samples, the maximum equivalent plastic strain is not located at the maximum stress triaxiality. The points seem to follow a unique tendency described in Ref. [67, 68] and shown in Figure 17(a). This needs to be confirmed for stress triaxiality levels $\eta>0.6$.

Similar to TA6V, the locus of maximum principal stress-equivalent plastic strain allowed for the construction of a limit line when $\sigma_{1}=1830 \mathrm{MPa}$. Such a criterion seems therefore also relevant for U500 (Figure 17(b)). Let us emphasize 


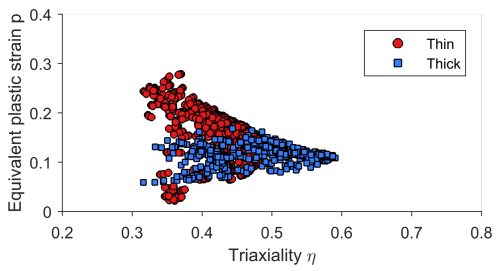

(a)

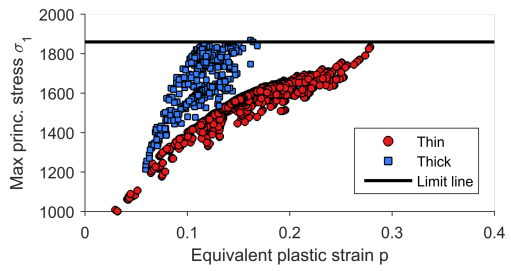

(b)

Fig. 17 Fracture loci $p(\eta)$ (a) and $\sigma_{1}(p)$ (b) for the elements in critical section of U500 samples for two different fracture planes

again that the results do depend on the plastic response and that the calculation should be performed with large strains.

For the evaluation of the critical damage indicator the fracture locus leads to a critical value of the order of $D_{i}=0.28$ for the damage indicator (Figure 18). This

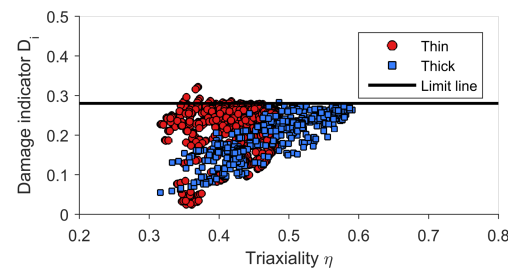

Fig. 18 Fracture locus for the damage indicator $D_{i}-$ stress triaxiality $\eta$ domain for the elements in critical sections of U500 samples

level was chosen as a lower bound to exclude some points that are believed to be outliers.

\section{Summary and Perspectives}

This paper was devoted to the failure analysis of TA6V and U500 alloys. The use of integrated DIC approaches enabled for the identification of failure criteria. A procedure was developed to analyze the mechanical fields in combination with the 
image of the fractured sample. The elements from the fractured surface are first extracted. The data of the extracted elements can then be analyzed to construct fracture loci. Several propositions for failure criteria from the literature were tested, namely, equivalent plastic strain - stress triaxiality and maximum principal stress - equivalent plastic strain. A damage indicator was also proposed, which weighs the equivalent plastic strain with a stress triaxiality function.

In a first step of the analysis the fractographies of TA6V and Udimet 500 alloys were presented. TA6V fails in a transgranular manner; no grains are visible. The fracture surface indicates that void nucleation and growth are the primary reason of final fracture. No indication of macroscopic damage was found before the occurrence of failure. For U500, the grains are visible and the fracture is intergranular. Therefore, in a first instance it is assumed that for both materials the material failure can be appropriately described by an initiation criterion. The onset of microscopic damage is neglected.

Other alternatives were studied [4] and it appears that the maximum principal stress criterion fits very well the data keeping in mind that any such analysis should consider plasticity calculations. In addition, it has to be noted that the fact that only one point for thick and thin samples reaches the limit value is attributed to the observation that once the failure initiates it propagates in a catastrophic manner. The analysis of all the data in time and space available from the I-DIC analyses was conducted to confirm that the criteria were not reached before and/or in other locations of the tested samples. For U500, two other fracture loci were consistent for thin and thick samples, namely, the equivalent plastic strain vs. stress triaxiality and a critical value of a damage indicator. 
The previous results, especially those concerning the maximum principal stress criterion, should be checked on other test cases, on sample geometries involving various states of stresses. Another improvement of the method would be the introduction of these fracture criteria in FE simulations. As shown herein for the two investigated materials, few elements reach the initiation condition. Moreover, no images of partially broken samples were available. The erosion of elements from the simulation or the use of cohesive elements would generate a virtual fracture surface. With additional procedures these predictions could be compared to the recorded images of the final fracture. One may also check whether once the failure initiates, it propagates in a catastrophic manner (as observed experimentally). In such simulations, special care should be exercised to make sure that the numerical predictions remain objective (i.e., mesh-insensitive).

Further, with an adapted I-DIC procedure, the crack can be recreated in the computer-generated images. Such a procedure would enable for the comparison of the fracture image with the I-DIC approach. The identification of plasticity and fracture could be performed simultaneously. This is similar to the ideas already proposed $[69,70]$ for which more local information was introduced in the identification functionals.

Another important point considers the identified constitutive model. For the thin and thick samples the average curves were not identical. First, the reason for such a difference needs to be further investigated. In this context, the sensitivity of the stress triaxiality fields to minimal changes in the identified law should be studied. Second, it should be possible to identify a unique constitutive model for all samples in a simultaneous identification. Together with the described improve- 
ments, the model identification for plasticity and fracture would then be one "big data" identification procedure.

Last, let us note that the experimental/numerical framework introduced herein is very generic. Other types of constitutive equations could have been investigated and other criteria probed (e.g., based on damage models). High-performance alloys were studied herein. Yet, the same analysis could have been performed on other types or classes of materials.

\section{Compliance with Ethical Standards}

The I-DIC code used herein is part of the Metil platform developed by Hugo Leclerc [71]. Dominik Lindner was supported by ANRT/CIFRE (grant number 2012-1519). This work was funded by SAFRAN Helicopter Engines. It is also a pleasure to acknowledge the support of BPI France ("DICCIT" project). The authors declare that they have no conflict of interest.

\section{References}

1. E. Robinson, Transactions of ASME 66, 373 (1944)

2. M. Mazière, J. Besson, S. Forest, B. Tanguy, H. Chalons, F. Vogel, European Journal of Mechanics - A/Solids 28(1), 36 (2009)

3. M. Mazière, J. Besson, S. Forest, B. Tanguy, H. Chalons, F. Vogel, European Journal of Mechanics - A/Solids 28(3), 428 (2009)

4. D. Lindner, Towards the fracture prediction of turbomachinery disks: A contribution of digital image correlation. PhD thesis, Université Paris-Saclay (2016)

5. D. Lindner, F. Mathieu, F. Hild, O. Allix, C. Ha Minh, O. Paulien-Camy, Journal of Applied Mechanics 82(7), 071014 (2015) 
6. P. Vacher, S. Dumoulin, F. Morestin, S. Mguil-Touchal, Proceedings of the Institution of Mechanical Engineers, Part C: Journal of Mechanical Engineering Science 213(8), 811 (1999)

7. M. Brunet, F. Morestin, Journal of Material Processing Technology 112(2-3), 214 (2001)

8. B. Wattrisse, A. Chrysochoos, J. Muracciole, M. Némoz-Gaillard, Experimental Mechanics 41(1), 29 (2001)

9. P.K. Rastogi, E. Hack (eds.), Optical methods for solid mechanics: a full-field approach (Wiley-VCH, Weinheim, 2012)

10. F. Hild, S. Roux, Experimental Mechanics 52(9), 1503 (2012)

11. F. Hild, S. Roux, Strain 42, 69 (2006)

12. H. Leclerc, J. Périé, S. Roux, F. Hild, Integrated digital image correlation for the identifcation of mechanical properties (Springer, Berlin (Germany), 2009), vol. LNCS 5496, pp. $161-171$

13. J. Réthoré, International Journal for Numerical Methods in Engineering 84(6), 631 (2010)

14. F. Mathieu, H. Leclerc, F. Hild, S. Roux, Experimental Mechanics 55(1), 105 (2015)

15. M. Abouridouane, Bruchverhalten von leichtmetallen unter impact-beanspruchung. Ph.D. thesis, RWTH Aachen, Aachen (2005)

16. H. Tresca, Comptes Rendus de l'Académie des Sciences (Paris) 59, 754 (1864)

17. R. von Mises, Nachrichten von der Gesellschaft der Wissenschaften zu Göttingen, Mathematisch-Physikalische Klasse 1913, 582 (1913)

18. R. Hill, Proceedings of the Royal Society of London A: Mathematical, Physical and Engineering Sciences 193(1033), 281 (1948)

19. W. Hosford, Journal of Applied Mechanics 39(2), 607 (1972)

20. P.W. Bridgman, Proceedings of the American Academy of Arts and Sciences 58(5), 163 $(1923)$

21. P. Bridgman, Collected Experimental Papers, vol. 1-7 (Harvard University Press, Cambridge, MA (USA), 1964)

22. P. Naghdi, F. Essenburg, W. Koff, Journal of Applied Mechanics 25(2), 201 (1958)

23. W. Lode, Zeitschrift für Physik 36(11), 913 (1926)

24. G.I. Taylor, H. Quinney, Philosophical Transactions of the Royal Society of London A: Mathematical, Physical and Engineering Sciences 230(681-693), 323 (1932) 
25. W. Osgood, Journal of Applied Mechanics 14, 147 (1947)

26. L. Bocher, P. Delobelle, Journal de Physique III 7, 1755 (1997)

27. E. Shiratori, K. Ikegami, Journal of the Society of Materials Science, Japan 16(165), 433 (1967)

28. E. Mönch, D. Galster, British Journal of Applied Physics 14(11), 810 (1963)

29. D. Hayhurst, The Journal of Strain Analysis for Engineering Design 8(2), 119 (1973)

30. D. Kelly, The Journal of Strain Analysis for Engineering Design 11(1), 1 (1976)

31. C. Morrison, in Techniques for Multiaxial Creep Testing, ed. by D. Gooch, I.M. How (Springer Netherlands, Dordrecht, 1986), pp. 111-126

32. A. Makinde, L. Thibodeau, K.W. Neale, Experimental Mechanics 32(2), 138 (1992)

33. J. Boehler, S. Demmerle, S. Koss, Experimental Mechanics 34(1), 1 (1994)

34. J.Y. Cognard, V. Feuardent, J.M. Virely, in Integrated Design and Manufacturing in Mechanical Engineering: Proceedings of the 1st IDMME Conference held in Nantes, France, 15-17 April 1996, ed. by P. Chedmail, J.C. Bocquet, D. Dornfeld (Springer Netherlands, Dordrecht, 1997), pp. 495-504

35. H.Y. Ko, R.F. Scott, Géotechnique 17(1), 40 (1967)

36. J. Pearce, in Stress-Strain Behaviour of Soils (Proceedings of the Roscoe Memorial Symposium), ed. by R. Parry (G.T. Foulis and Co., Henley-on Thames, 1971), pp. 330-339

37. S. Shima, K. Mimura, International Journal of Mechanical Sciences 28(1), 53 (1986)

38. J. Lanier, in Geomaterials: Constitutive Equations and Modelling, ed. by F. Darve (Elsevier Applied Sciences, London (UK), 1990), pp. 7-26

39. S. Calloch, D. Marquis, International Journal of Plasticity 15, 521 (1999)

40. S. Calloch, C. Bouvet, F. Hild, C. Doudard, C. Lexcellent, (SPIE Proceedings, 2002), vol. 4537, pp. $83-86$

41. M. Petkovski, R.S. Crouch, P. Waldron, Experimental Mechanics 46(3), 387 (2006)

42. K. Nakazima, T. Kikuma, K. Hasuka, Study on the formability of steel sheets. Tech. Rep. 264, Yawata (1968)

43. Z. Marciniak, K. Kuczyński, T. Pokora, International Journal of Mechanical Sciences $\mathbf{1 5}(10), 789(1973)$

44. S. Keeler, SAE Technical Paper 650535 pp. 1-9 (1965)

45. G. Goodwin, SAE Technical Paper 680093 pp. 1-8 (1968) 
46. S. Keeler, SAE Technical Paper 680092 pp. 1-9 (1968)

47. P. Vacher, A. Haddad, R. Arrieux, CIRP Annals - Manufacturing Technology 48(1), 227 (1999)

48. F. Breutinger, Verformungsverhalten und Verformungskinetik von Titan technischer Reinheit und der Titanlegierung TiAl6v4 im Bereich niedriger homologer Temperaturen von 0, $22\left(150^{\circ} \mathrm{C}\right)$ bis $0,48\left(650^{\circ} \mathrm{C}\right)$. Ph.D. thesis, Universität Erlangen-Nürnberg (2006)

49. S. Sajjadi, H. Elahifar, H. Farhangi, Journal of Alloys and Compounds 455(1-2), 215 $(2008)$

50. J.R. Davis, Nickel, cobalt, and their alloys. ASM specialty handbook (ASM International, Materials Park, OH, 2000)

51. N. Lambert, J.M. Drapier, in International Symposium on Structural Satbility of Superalloys (1968)

52. F. Hild, S. Roux, in Optical Methods for Solid Mechanics. A Full-Field Approach, ed. by P. Rastogi, E. Hack (Wiley-VCH, Weinheim (Germany), 2012), pp. 183-228

53. A. Barré de Saint-Venant, Comptes Rendus de l'Académie des Sciences (Paris) 70, 473 $(1870)$

54. E. Beltrami, Il Nuovo Cimento (1877-1894) 18(1), 145 (1885)

55. M. Huber, Czasopismo Techniczne, Lwów 15 (1904)

56. H. Hencky, Zeitschrift für Angewandte Mathematik und Mechanik 4(4), 323 (1924)

57. C.A. Coulomb, Mémoires de mathématique \& de physique, présentés à l'Académie Royale des Sciences par divers savans 7, 343 (1773)

58. W.M. Rankine, Philosophical Transactions of the Royal Society of London 147, 9 (1857)

59. F. McClintock, Journal of Applied Mechanics 35(2), 363 (1968)

60. J. Rice, D. Tracey, Journal of the Mechanics and Physics of Solids 17, 201 (1969)

61. J. Lemaitre, Journal of Engineering Materials and Technology 107(83-89) (1985)

62. J. Lemaitre, A Course on Damage Mechanics (Springer-Verlag, Berlin (Germany), 1992)

63. G.Z. Voyiadjis (ed.), Handbook of Damage Mechanics (Springer, New York, NY (USA), 2014)

64. T. Morgeneyer, T. Taillandier-Thomas, L. Helfen, T. Baumbach, I. Sinclair, S. Roux, F. Hild, Acta Materialia 69, 78 (2014)

65. O. Allix, P. Feissel, H. Nguyen, Engineering Computation 22(5-6), 487 (2005) 
66. P. Feissel, O. Allix, Computer Methods in Applied Mechanics and Engineering 196(13), 1968 (2007)

67. Y. Bai, T. Wierzbicki, International Journal of Plasticity 24(6), 1071 (2008)

68. J. Papasidero, V. Doquet, D. Mohr, International Journal of Solids and Structures 69-70, $459(2015)$

69. S. Roux, J. Réthoré, F. Hild, Journal of Physics D: Applied Physics 42(21), 214004 (2009)

70. T.S. Cao, E. Maire, C. Verdu, C. Bobadilla, P. Lasne, P. Montmitonnet, P.O. Bouchard, Computational Materials Science 84, 175 (2014)

71. H. Leclerc, in 8e Colloque National en Calcul des Structures (2007) 